\title{
The sodium/iodide symporter NIS is a transcriptional target of the p53-family members in liver cancer cells
}

\author{
F Guerrieri ${ }^{1,2}$, S Piconese ${ }^{3}$, C Lacoste ${ }^{4,5}$, V Schinzari ${ }^{1,2}$, B Testoni ${ }^{1,2}$, Y Valogne ${ }^{4,5}$, S Gerbal-Chaloin ${ }^{6}$, D Samuel ${ }^{4,5}$, C Bréchot ${ }^{4,5}$, \\ J Faivre $^{\star, 4,5,7}$ and M Levrero ${ }^{*, 1,2,3}$
}

Thyroid iodide accumulation via the sodium/iodide symporter (NIS; SLC5A5) has been the basis for the longtime use of radio-iodide in the diagnosis and treatment of thyroid cancers. NIS is also expressed, but poorly functional, in some non-thyroid human cancers. In particular, it is much more strongly expressed in cholangiocarcinoma (CCA) and hepatocellular carcinoma (HCC) cell lines than in primary human hepatocytes (PHH). The transcription factors and signaling pathways that control NIS overexpression in these cancers is largely unknown. We identified two putative regulatory clusters of p53-responsive elements (p53REs) in the NIS core promoter, and investigated the regulation of NIS transcription by p53-family members in liver cancer cells. NIS promoter activity and endogenous NIS mRNA expression are stimulated by exogenously expressed p53-family members and significantly reduced by member-specific siRNAs. Chromatin immunoprecipitation analysis shows that the p53-REs clusters in the NIS promoter are differentially occupied by the p53-family members to regulate basal and DNA damageinduced NIS transcription. Doxorubicin strongly induces p53 and p73 binding to the NIS promoter, leading to an increased expression of endogenous NIS mRNA and protein in HCC and CCA cells, but not in PHH. Silencing NIS expression reduced doxorubicin-induced apoptosis in HCC cells, pointing to a possible role of a p53-family-dependent expression of NIS in apoptotic cell death. Altogether, these results indicate that the NIS gene is a direct target of the p53 family and suggests that the modulation of NIS by DNA-damaging agents is potentially exploitable to boost NIS upregulation in vivo.

Cell Death and Disease (2013) 4, e807; doi:10.1038/cddis.2013.302; published online 19 September 2013

Subject Category: Cancer

The solute carrier family 5 member A5 SLC5A5, commonly referred to as the sodium/iodide symporter (NIS), is a transmembrane glycoprotein that mediates active iodide uptake into thyroid follicular cells and thereby has a crucial role in the biosynthesis of thyroid hormones. ${ }^{1,2}$ Expression of a functional NIS protein was also found in several other normal tissues, such as stomach, salivary glands and lactating mammary gland. ${ }^{3-5}$

NIS-mediated ability of thyroid cancer cells to accumulate iodide is exploited for diagnostic nuclear imaging and radioiodine therapy. ${ }^{6,7}$ However, NIS expression in thyroid cancers decreases as the cancer cell differentiation decreases, ${ }^{5}$ rendering non-differentiated thyroid cancers refractory to radioiodine therapy. In the stomach NIS expression is generally suppressed, during carcinogenesis. ${ }^{8}$ A NIS expression was found in a number of other non-thyroid cancers, among which those of the breast and liver.,9,10 In breast cancer cells, NIS expression was stimulated by retinoic acid (RA), estrogen and glucocorticoids, and it was induced through the Rac1/p38b mitogen-activated protein kinase
(MAPK), cAMP and phosphoinositide-3 kinase (PI3K)-signaling pathways. ${ }^{11-14}$ In liver cancers, NIS was expressed in all the cholangiocarcinoma (CCA) and in a small proportion of the hepatocellular carcinoma (HCC), studied. ${ }^{9}$ NIS expression was detected in the precancerous stages of liver cancer in a rat model of HCC, and further amplified along clonal tumor progression. $^{9}$ In a majority of NIS-expressing non-thyroid cancers, NIS is largely retained intracellularly with a low cell surface density, and showed little or no iodide uptake activity. ${ }^{15-17}$ The role of NIS in carcinogenesis and the function of intracellular NIS in normal and neoplastic tissues have recently been clarified by discovering a role of NIS in cell migration and invasion without ion transport being involved. ${ }^{18}$

The transcriptional regulation of NIS expression is complex. It involves different regulatory regions and the activity of both thyroid-enriched and ubiquitous transcription factors. ${ }^{19,20}$ An upstream enhancer (NUE), located in human cells between -9470 and -9046 relative to ATG, mediates NIS thyroid specific expression. ${ }^{19}$ The hNUE is strongly responsive to TSH stimulation and contains putative cis-elements for

\footnotetext{
${ }^{1}$ LEA INSERM U785, Department Internal Medicine, DMISM, Sapienza University, Rome, Italy; ${ }^{2}$ Laboratory of Gene Expression, Fondazione A. Cesalpino, Rome, Italy; ${ }^{3}$ Laboratory of Cellular Immunology-DMISM, Sapienza University, Rome, Italy; ${ }^{4}$ INSERM U785, Centre Hépatobiliaire, Hôpital Paul-Brousse, Villejuif, France; ${ }^{5}$ Faculté de Médecine Bicêtre, Université Paris-Sud, Bicêtre, France; ${ }^{6}$ INSERM U632, Institut de Recherche en Biothérapie, Montpellier, France and ${ }^{7}$ Department of Hematology and Tumor Biology, Paul-Brousse Hospital, Villejuif, France

*Corresponding author: J Faivre, INSERM U785, Centre Hépatobiliaire, Hôpital Paul-Brousse, 14 Avenue, Paul Vaillant-Couturier, Villejuif 94800 , France. Tel: +33 01 45596075; Fax: +33 01 45593857; E-mail: jamila.faivre@ inserm.fr

or M Levrero, LEA INSERM U785, Department Internal Medicine, DMISM, Sapienza University, Viale del Policlinico 155, Rome 00161 , Italy. Tel: +39 0649970892 ; Fax: +39 06 49383333; E-mail: massimo.levrero@uniroma1.it

Keywords: NIS; p53; p63; p73; DNA damage

Abbreviations: NIS, sodium/iodide symporter; p53-RE, p53-responsive element; PHH, primary human hepatocytes; CCA, cholangiocarcinoma; HCC, hepatocellular carcinoma; MAPK, mitogen-activated protein kinase; NUE, NIS, upstream enhancer

Received 21.3.13; revised 24.6.13; accepted 01.7.13; Edited by G Melino
} 
the Pax-8 and Nkx2.1 transcription factors, which are both required for thyroid development and differentiation. ${ }^{21} \mathrm{Pax}-8$ sites co-operate with an adjacent cAMP-responsive element to ensure full hNUE activity ${ }^{22-24}$ and mediate the transcriptional repression of hNIS expression by the PBF (pituitary tumor-transforming gene (PTTG)-binding factor) proto-oncogene. ${ }^{25}$ The hNIS core promoter region spans between -475 and -393 relative to ATG and contains a TATA-like motif and a GC-box. Binding of Sp1 and a 'Sp1-like protein' to the GC-box is required for a basal activity of NIS promoter. ${ }^{26}$ A RA-response element located at position -1375 relative to NIS start codon mediates the activation of NIS expression by tRA in human follicular thyroid carcinoma cells. ${ }^{27}$ NIS regulation by retinoids in breast cancer cells is effected through a downstream intronic enhancer, which binds RAR $\alpha$ and $\mathrm{RXR} .{ }^{28-33}$ Moreover, the cardiac homeobox transcription factor Nkx2.5, which is induced upon tRA stimulation, binds two cis-acting elements in the hNIS promoter located at -446 and -154 relative to $A T G .{ }^{34}$

NIS upregulation in a large spectrum of cancers with different genetic backgrounds suggests the involvement of molecular factors common to these cancers, which remain, however, to be identified. We investigated the possible role of the p53 gene family in the transcriptional regulation of NIS expression in normal and neoplastic liver cells. In silico analyses revealed that the proximal regulatory region of human NIS gene contains numerous responsive elements to p53. Here, we show that NIS gene is a direct target of the p53-family proteins and that DNA damage triggers NIS gene activation through a differential binding of p53 and p53-related proteins to NIS proximal promoter.

\section{Results}

NIS is a transcriptional target of the p53-family members in liver cells. A previous study of NIS expression in human primary liver cancers revealed significant levels of NIS in all the tumor cholangiocytes and in a small proportion of the tumor hepatocytes studied, whereas all the tumor hepatocytes expressed NIS in the DEN (diethyl nitrosamine) rat model of $\mathrm{HCC}^{9}$ The reasons for such differences in NIS expression are unknown. To identify cell models appropriate for NIS transcription studies, we investigated by real-time PCR the expression levels of NIS mRNA in well-characterized human HCC and CCA cell lines. Figure 1a shows the un-stimulated NIS mRNA levels in Hep3B, HepG2, HuH7 (human HCC) and CCSW1, CCLP1 (human CCA) cells. The Hep3B cell line is p53 null; the HepG2 and CCSW1 liver cancer cell lines have a wild-type $p 53$ gene, while the $\mathrm{HuH} 7$ and CCLP1 cell lines carry p53 point mutations. ${ }^{35-38}$ All cancer cell lines except for the p53 null Hep3B displayed a clear NIS mRNA expression, whether they harbored a wildtype or a mutated p53. In agreement with previous studies of NIS expression in normal liver, ${ }^{9}$ primary human hepatocytes $(\mathrm{PHH})$ display only a very weak NIS expression (Figure 1a). NIS protein was detected in the HuH7, CCLP1 (Figure 1b) and HepG2 (Figure 1c) cell lines, and not in $\mathrm{PHH}$ or Hep3B cells Figure 1b). Note that the NIS protein is almost completely accumulated at the cell surface in the HepG2 cell line (Figure 1c). In silico analysis of the NIS regulatory region $(-5000 /+1500$ relative to human NIS gene transcription start site (TSS)) using the Genomatix package (www.genomatix.de) (cutoff score of $80 \%$ ) allowed us to identify several p53-responsive elements grouped into two putative regulatory clusters called ' $A$ ' and ' $B$ ' (p53-responsive element; p53RE) (Figure 1d). Cluster A includes three p53 sites located between -1600 and $-2000 \mathrm{bp}$ and corresponds to the p63-binding region previously identified by Testoni et al. ${ }^{39}$ Cluster $\mathrm{B}$ resides within the NIS proximal promoter region ${ }^{20}$ and includes five p53REs located between -400 and $-700 \mathrm{bp}$. Moreover, we found six conserved SP1-binding sites in the human NIS proximal promoter, one in cluster $A$ and many additional ones either downstream the ATG or upstream cluster A. We performed chromatin immunoprecipitation (ChIP) assays in $\mathrm{PHH}$, HepG2 cells and CCSW1 cells to study the binding of the p53-family members to clusters A and B of the NIS gene. In the HepG2 and CCSW1 cancer cell lines, all the p53-family members showed a low binding to cluster A (Figure 1e). Cluster B displayed an overall higher occupancy by the p53-family members. A particularly strong binding to cluster B was found for p53 and p73 in HepG2 cells, and for p63 and p73 in CCSW1 cells (Figure 1e). In $\mathrm{PHH}$, a low binding to both clusters was found for p53, p73 and, even more so, p63. Control PCR reactions using distant NIS primers did not amplify any anti-p53, anti-p63 or anti-p73 ChIPed products (data not shown). Furthermore, ChIP analysis of SP1 occupancy showed an uniform recruitment to both cluster A and cluster B in all three cell types (Supplementary Figure S1 and data not shown), suggesting that the differential recruitment of p53, p63 and p73 according to cluster and cell type might have functional relevance.

p53-family members activate NIS expression in liver cancer cells. We investigated the ability of the p53-family members to regulate NIS promoter activity. HCC (HepG2, Huh7 and Hep3b) and CCA (CCSW1 and CCLP1) cell lines were co-transfected with a NIS promoter luciferase (NIS-Luc) reporter plasmid containing the $-2000 /+375$ sequence of NIS promoter and encompassing clusters $A$ and $B$ either alone or together with expression plasmids encoding p53, $p 63 \alpha$ and $p 73 \alpha$. Protein levels of exogenously expressed p53, p63 $\alpha$ and $p 73 \alpha$ are shown in Supplementary Figure S2. Luciferase activity assayed $24 \mathrm{~h}$ after transfection and normalized for transfection efficiency using the dual-luciferase reporter system was expressed as fold induction over the control. Exogenously expressed $\mathrm{p} 63 \alpha$ and $\mathrm{p} 73 \alpha$ elicited an important stimulation of the NIS-Luc reporter plasmid in all the HCC cell lines studied (Figure 2a). Exogenous p63 $\alpha$ and p73 $\alpha$ both activated the NIS gene promoter in the CCLP1 CCA cell line, whereas only $p 73 \alpha$ had a significant stimulatory effect in CCSW1 cells. Exogenous p53 activated the NIS gene promoter in Huh7 and Hep3B cells, but had no significant effect in HepG2, CCLP1 or CCSW1 cells. The expression level of the endogenous NIS mRNA was strongly increased in HepG2 cells after transfection with the expression vectors for $\mathrm{p} 73 \alpha$ (about 10x increase) and to a lesser extent with the $p 63 \alpha$ vector (Figure $2 b$ ). The ability of exogenously expressed $p 63 \alpha$ and $p 73 \alpha$ to increase NIS 


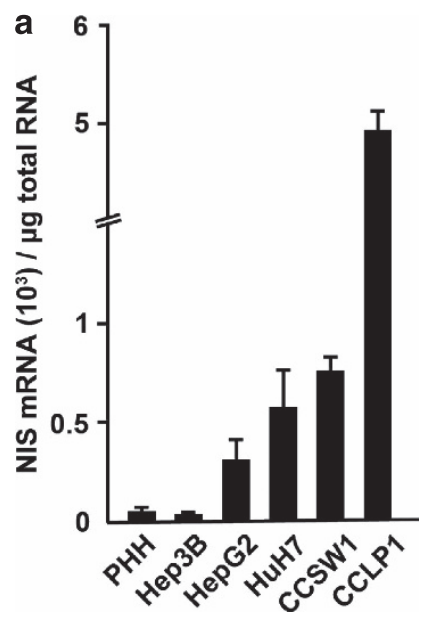

b

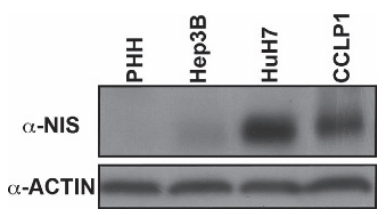

C
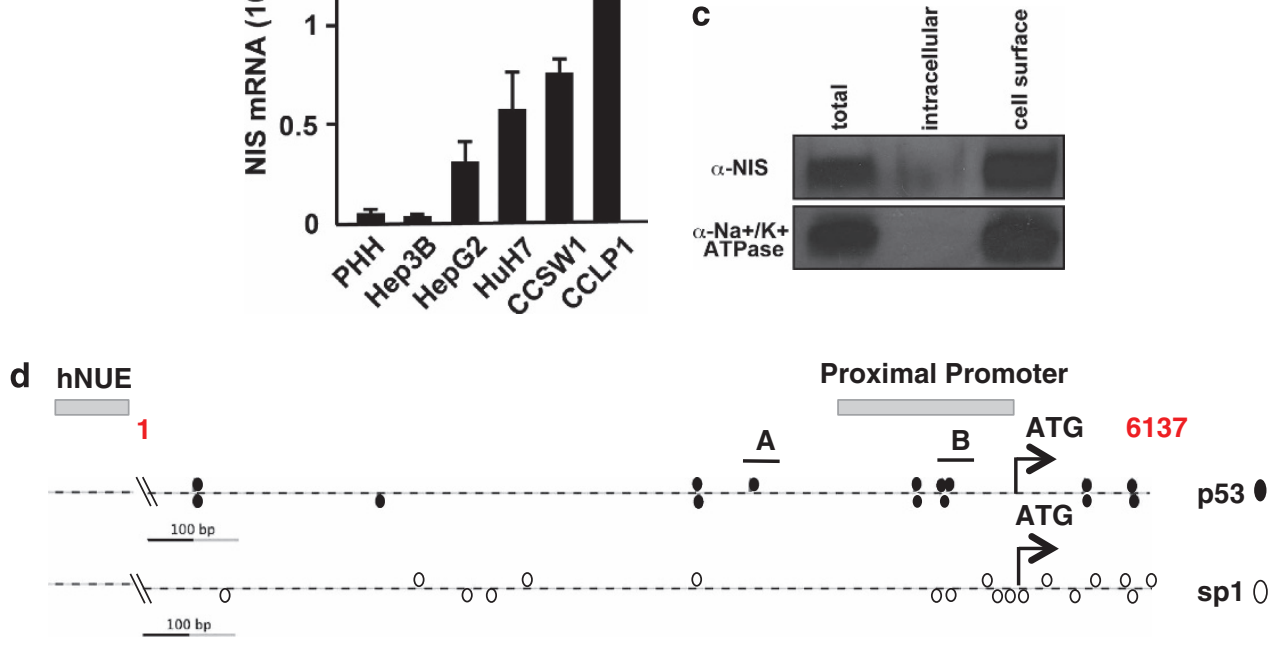

e
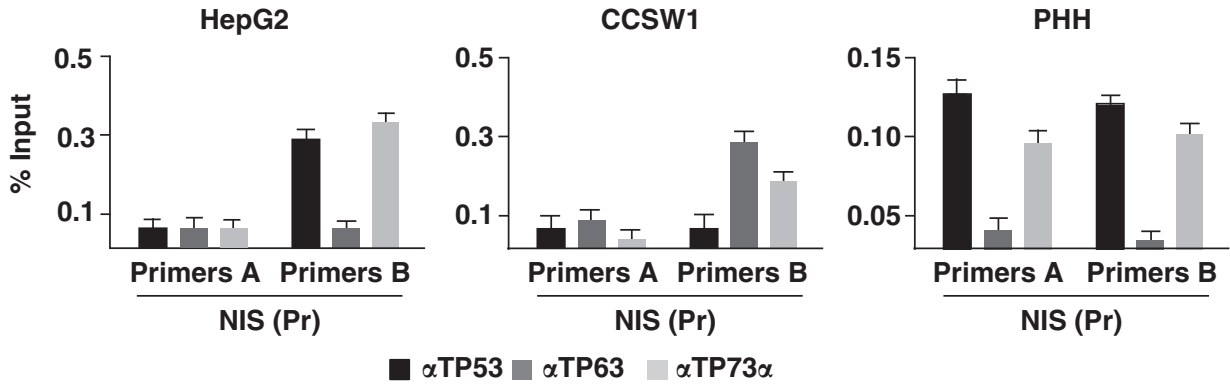

Figure 1 NIS is a target of the p53-family members. (a) Real-time RT-PCR for NIS mRNA in primary hepatocytes (PHH), hepatocellular carcinoma (Hep3B, HuH7 and HepG2) and CCA (CCSW1 and CCLP1) cell lines. Data are expressed as mean values \pm S.E.M. of three independent experiments performed in duplicate. (b) Anti-NIS immunoblot of total protein extracts from the indicated cells. (c) Anti-NIS and anti-Na ${ }^{+}-\mathrm{K}^{+}$ATPase immunoblots after biotinylation and isolation of cell surface proteins in HepG2 cells. (d) Schematic representation of the human NIS proximal regulatory region $(-1000 /+5000$ relative to NIS gene TSS sequence). In silico analysis was performed using the Genomatix package (cutoff score of $80 \%$ ). Solid circles: p53-binding sites. Open circles: Sp1 consensus sites. Hatched rectangles: human NIS upstream enhancer (hNUE) and NIS proximal promoter regions. Solid rectangles: location of the PCR products obtained with the two ChIP primer pairs called A and B. (e) ChIP qPCR analysis of the interaction between NIS promoter and the p53-family proteins. Cross-linked chromatin from HepG2 and CCSW1 cells and PHHs (PHH) was immunoprecipitated with specific anti-p53 ( $\alpha$ TP53), anti-p63 ( $\alpha$ TP63), and anti-p73 $\alpha(\alpha$ TP73 $\alpha$ ) antibodies, or the relevant IgG controls, and then analyzed by real-time qPCR using the NIS promoter ( $\mathrm{Pr}$ ) A or B primer pairs. Control reactions using distant primers did not amplify anti-p53, anti-p63 or anti-p73 $\alpha$ ChIPped products (data not shown). Data are means \pm S.D. from three independent experiments

protein levels in HepG2 cells was confirmed by immunoblotting (Figure 2c). In CCSW1 cells, both p63 $\alpha$ and $p 73 \alpha$ induced a significant increase in NIS mRNA expression (Figure 2b). The role of p53-family members in the transcriptional regulation of NIS expression was further confirmed by the strong effect of the abrogation of p53, p63 and p73 expression by specific siRNAs (Supplementary Figure S3) on NIS promoter activity (Figure 2d). Altogether, these results clearly imply that the p53-family members are involved in the regulation of NIS transcription in liver cancer cell lines.

Doxorubicin increases NIS expression in liver cancer cells. The p53 tumor suppressor protein is a potent inducer of tumor cell death, which is activated in response to DNA damage and DNA-damaging agents through specific posttranslational modifications. There is an increasing evidence that the p53 paralogs p73 and p63 are also activated in response to DNA damage and have an important role in the chemosensitivity of tumor cells. ${ }^{40}$ The genotoxic drug doxorubicin is known to induce an accumulation of p53-family proteins in numerous different cell types. ${ }^{40}$ As NIS is a direct target of the p53-family members, we investigated the effects of doxorubicin on NIS promoter activity and expression. The tumor cell lines HepG2, Huh7, Hep3B and CCSW1 were transfected with the $-2000 /+375$ NIS-Luc reporter plasmid and exposed to an apoptotic dose $(2 \mu \mathrm{M})$ of doxorubicin. It was found that NIS promoter activity 

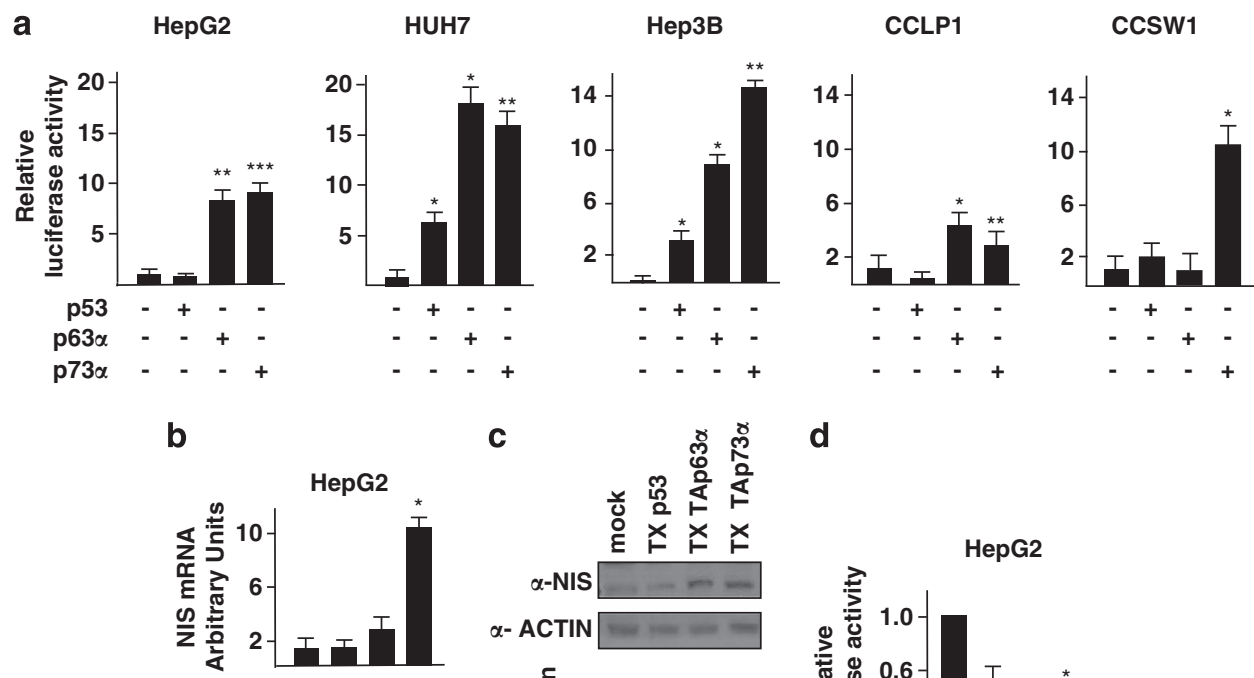

c

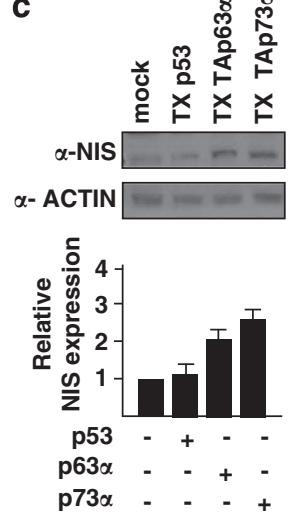

d

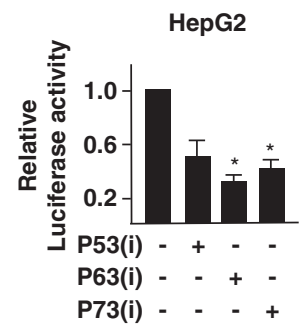

Figure 2 p53-family members activate transcription of NIS promoter in liver cancer cell lines. (a) The indicated HCC (HepG2, HUH7 and Hep3B) and CCA (CCSW1 and CCLP1) cell lines were co-transfected with $300 \mathrm{ng}$ of the $-2000 /+375 \mathrm{NIS}$ promoter luciferase construct and either p53, p63 $\alpha$ or p $73 \alpha$ expression vectors Luciferase activity was assayed $24 \mathrm{~h}$ after transfection and expressed as fold induction relative to the control after normalization for transfection efficiency using the dual-luciferase assay system. Histograms show the means of at least three experiments performed in quadruplicate. Bars indicate S.D. (b) NIS mRNAs levels were determined by real-time qPCR using NIS-specific primers in HepG2 and CCSW1 cells transfected with the $-2000 /+375$ NIS promoter luciferase construct and the indicated expression vectors. Results are expressed as fold induction relative to the mock-transfected controls after normalization towards endogenous human $\beta$-actin mRNAs (beta actin FAM Probe Roche) mRNAs. Data are means \pm S.D. from at least three independent experiments performed in duplicate. (c) Anti-NIS immunoblot of HepG2 cells transfected with $p 53, p 63 \alpha$ or p $73 \alpha$ expression vectors (upper panel). $\alpha$-Actin protein levels were used to normalize equal loadings from lysate samples. Histograms (lower panel) represent the means of three independent experiments. Bar, S.D. (d) HepG2 cells were co-transfected with the $-2000 /+375$ NIS luciferase construct and $100 \mathrm{nM}$ of p53, p63 and p73 small interfering RNAs or a control siRNA smart pool. Luciferase activity was determined as in (a). Data are means \pm S.D. of at three independent experiments performed in duplicate. $P$-values were determined using the two-tails Student's $t$-test. ${ }^{*} P<0.05 ;{ }^{* \star} P<0.01$; ${ }^{* \star *} P<0.001$

was strongly induced ( $\sim 50$-fold) by doxorubicin in HepG2 and, to a lesser extent (5-fold), in CCSW1 cells (Figure 3a). NIS upregulation diminished by $50 \%$ upon siRNA interference for p53 (Supplementary Figure S4). Endogenous NIS mRNA expression was likewise upregulated by doxorubicin in HepG2 and CCSW1 cells (Figure 3b). NIS promoter activity and NIS mRNA levels were strongly induced in response to doxorubicin also in Huh7 cells harboring a mutant p53 (Figures $3 a$ and b). As shown in Supplementary Figure S5, NIS activation in response to doxorubicin was accompanied in Huh7 cells by a sharp recruitment of TAp73 $\alpha$ on the NIS promoter, whereas p63 occupancy was not affected. No significant NIS induction was observed in Hep3B cells, which are p53 null and do not express $p 73^{41,42}$ (Figures $3 a$ and b). PHHs also did not upregulate NIS expression in response to doxorubicin (Figure $3 b$ ). A strong induction of NIS promoter activity was also observed in HepG2 cells exposed to etoposide and cisplatin (Supplementary Figure S6). Altogether, these observations demonstrate that NIS is activated by $\mathrm{p} 53$ and p53-related proteins at the transcriptional level in response to DNA damage in liver cancer cells.
The p53-family proteins are differentially recruited to the NIS proximal promoter in response to doxorubicin. Next, we compared the binding of the p53-family members with the NIS promoter region in liver cells before and after doxorubicin treatment. ChIP analysis was performed in HepG2, CCSW1 and PHH cells either exposed to $2 \mu \mathrm{M}$ doxorubicin or left untreated. Figure 4 shows the real-time PCR quantification of $p 53, p 63$ and $p 73$ recruitment to region A (upper panels) and region B (lower panels) of the NIS promoter, expressed as relative enrichment factor between doxorubicin-treated and untreated cells. Control PCR reactions using distant NIS primers did not amplify any anti-p53, anti-p63 or anti-p73 ChIP-ed DNA (data not shown). No increase in the binding of the p53-family members to region $A$ following doxorubicin stress was observed in HepG2 and CCSW1 cells (Figure 4, upper panels). In contrast, p53 and p73 (but not p63) were actively recruited to region $B$ in response to doxorubicin in both cell lines. In $\mathrm{PHH}$, doxorubicin did not induce any significant recruitment of p53 and p73 to region $A$ and caused only a slight increase in the recruitment of $p 63$ and $p 73$ to region $B$. These data demonstrate a differential recruitment of the p53-family 

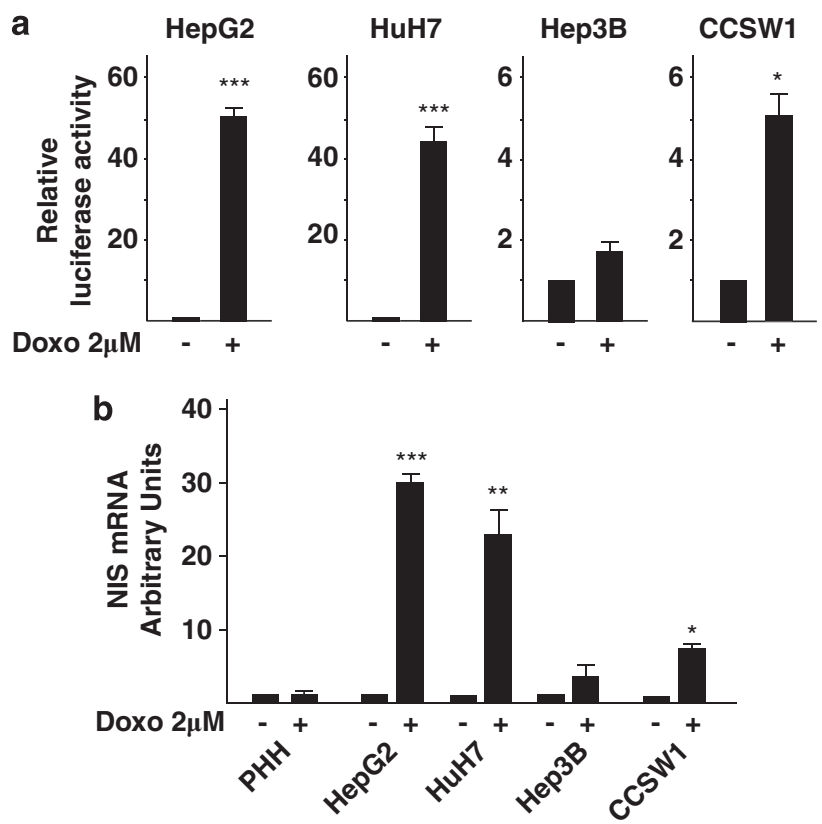

Figure 3 NIS gene promoter activity is induced by doxorubicin in liver cancer cell lines. (a) Luciferase activity normalized for transfection efficiency and expressed as fold induction with respect to control. The indicated cell lines were transfected with the $-2000 /+375$ NIS luciferase construct and exposed for $24 \mathrm{~h}$ to doxorubicin (doxo $2 \mu \mathrm{M}$ ). Luciferase activity was assayed $30 \mathrm{~h}$ after transfection and expressed as in Figure 2a. Data are means \pm S.D. of at least three experiments performed in duplicate. (b) NIS mRNA levels in untreated (NT) or doxorubicintreated (DOXO) cells were analyzed and results expressed as in Figure 2b. Data are means \pm S.D. of at least three experiments performed in duplicate. $P$-values were determined using the two-tails Student's $t$-test. ${ }^{*} P<0.05$; ${ }^{* *} P<0.01$; ${ }^{* \star} P<0.001$ proteins to the NIS promoter in response to a doxorubicininduced stress and suggest that these transcription factors, mostly p53 itself, specifically regulate region B of the NIS promoter in response to DNA damage.

NIS contributes to DNA damage-induced apoptosis in HepG2 cells. The function of NIS over-expressed in many cancers is unknown. In this study, we have shown that DNA damage triggers a transcriptional activation of NIS in liver cancer cells that is mediated by p53 and p53-related proteins, and suggests that NIS could be involved in DNA damage-induced apoptosis. We therefore investigated the role played by an accumulation of NIS in the response of liver cancer cells to doxorubicin-induced apoptosis. Exposure to $2 \mu \mathrm{M}$ doxorubicin induced both apoptosis, as evidenced by detection of poly (ADP-ribose) polymerase (PARP)-cleaved fragments, and accumulation of mature $97 \mathrm{kDa}$ NIS species in HepG2 cells (Figure 5a). Similar results were obtained in CCSW1 cell (Supplementary Figure S7). Flow cytometry analysis of intact living cells exposed to doxorubicin showed a statistically significant, although moderate, increase of NIS expression both at the cell surface and intracellularly in HepG2 cells (Figure 5b). A small but consistent and significant increase of NIS protein expression at the cell surface was also detected in CCSW1 CAA cells (Supplementary Figure S8), but not in the p53 null cell line Hep3b (Supplementary Figure S8). We also studied the response of HepG2 cells to doxorubicin after knockdown of endogenous NIS protein expression by specific siRNAs (Supplementary Figure S9). We found that NIS silencing caused a reduction of PARP cleavage (Figure $5 \mathrm{c}$ ) and

a

HepG2

ccsw1

PHH
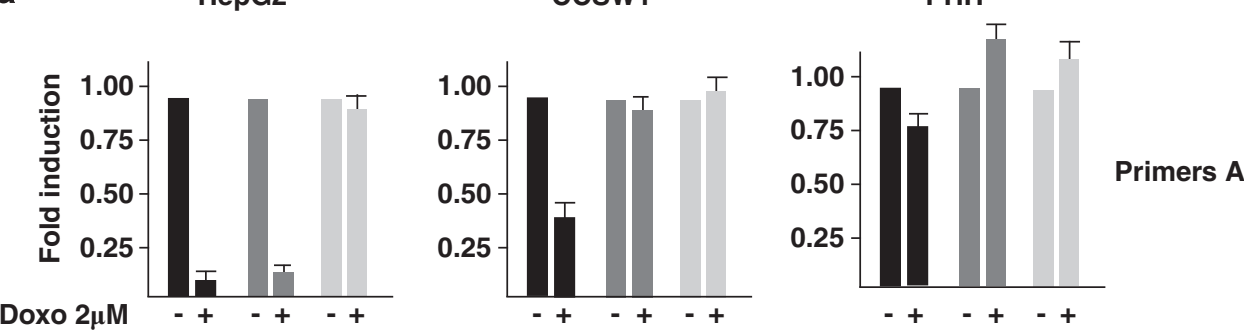

b
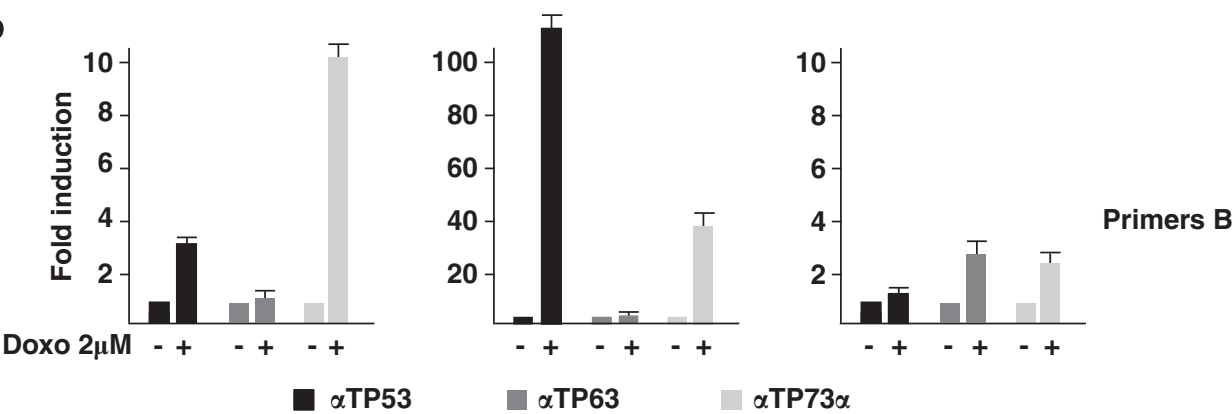

Figure 4 p53 and p73 are actively recruited to NIS proximal promoter in response to doxorubicin in liver cancer cells. Cross-linked chromatin from untreated and doxorubicin-treated ( $2 \mu \mathrm{M} ; 24 \mathrm{~h})$ HepG2 cells, CCSW1 cells and PHHs was immune-precipitated with the relevant control IgG or specific anti-p53 ( $\alpha$ TP53), anti-p63 ( $\alpha$ TP63) and anti-p73 $\alpha(\alpha$ TP73 $\alpha)$ antibodies and analyzed by real-time PCR with the A and B pairs of NIS promoter selective primers. Control reactions using distant primers did not amplify anti-p53, anti-p63 or anti-p73 $\alpha$ ChIPed products (data not shown). Histograms represent the means of three independent experiments. Bar, S.D. 


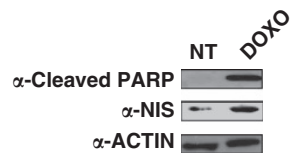

b

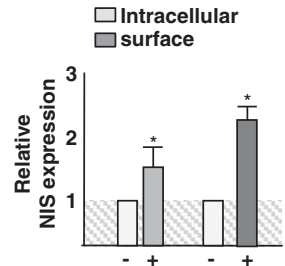

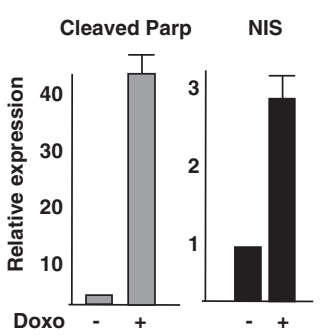

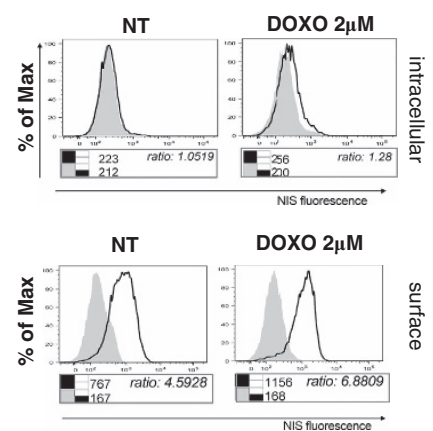

d

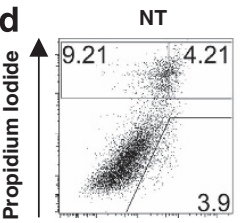

Doxo DOXO iNIS
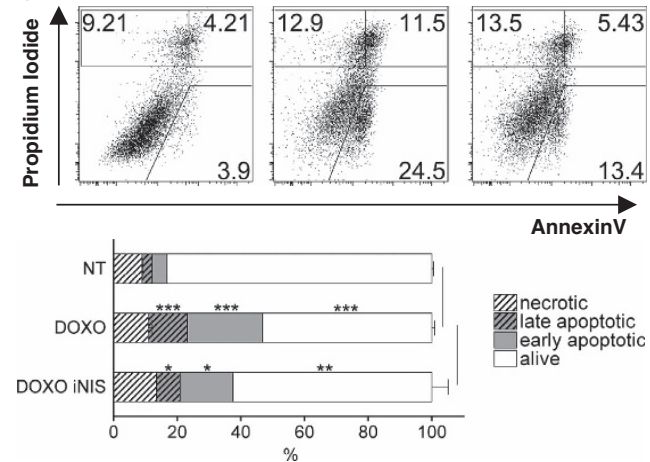

AnnexinV

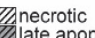
late apoptotic early apoptotic alive
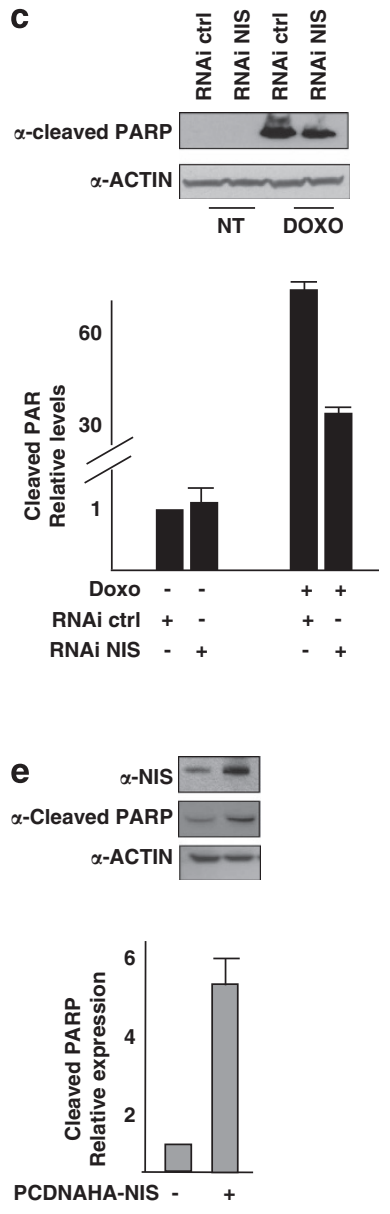

Figure 5 NIS silencing reduces DNA damage-induced apoptosis in HepG2 cells. (a) Whole-cell extracts from untreated and doxorubicin-treated ( $2 \mu$ M; $24 \mathrm{~h}$ ) HepG2 cells were analyzed by immunoblot using anti-PARP- and anti-NIS-specific antibodies. The caspase cleaved $89 \mathrm{kDa}$ fragment of PARP and the $97 \mathrm{kDa}$ major NIS glycoprotein species are revealed. $\alpha$-Actin protein levels were used to normalize equal loadings from lysate samples. (b) Flow cytometry analysis of cell surface (left) and intracellular (right) NIS expression in untreated (NT) or doxorubicin-treated (DOXO) $(2 \mu \mathrm{M}, 24 \mathrm{~h})$ HepG2 cells. Top panel: NIS cell surface expression is presented as the ratio of the mean fluorescence intensity (MFI) of samples stained with anti-NIS primary and secondary antibodies to that of samples only stained with the secondary antibody. Data are means \pm S.D. of at least three independent experiments performed in duplicate. Bottom panel: histograms of cell fluorescence intensity in samples stained with anti-NIS primary and secondary antibodies (solid lines) and samples stained only with the secondary antibody (gray areas). At least 5000 events were acquired in the selected (live cells/singlets) gate for each sample. (Inset) MFI values of the histograms. (c) Cleaved PARP immunoblot in HepG2 cells transfected with NIS small interfering RNA or a control siRNA smart pool and either left untreated (NT) or exposed to $2 \mu \mathrm{M}$ Doxorubicin for $24 \mathrm{~h}$ (DOXO). $\alpha$-Actin protein levels were used to normalize equal loadings from lysate samples. Histograms (lower panel) represent the means of three independent experiments. Bar, S.D. (d) Flow cytometry quantification of early (annexin $\mathrm{V}+\mathrm{ve} \mathrm{PI}+\mathrm{ve}$ (Propidium lodide, subG1 cells)) and late (annexinV + ve and PI + ve) apoptotic HepG2 cells either left untreated or exposed to $2 \mu \mathrm{M}$ doxorubicin for $18 \mathrm{~h}$, transfected with control siRNA pool or specific siNIS. (Upper panel) Dot plots of untreated (NT), doxorubicin-treated (DOXO), siNIS-transfected and doxorubicin-treated (DOXO iNIS) HepG2 cells. (Numbers) Percentage of PI, annexin V and annexin V + PI-positive cells. (Right panel) Results from three independent experiments. Horizontal bars: mean values of necrotic (annexin V - ve PI + ve), late apoptotic (annexin V + ve PI + ve) and early apoptotic (annexin V + ve PI-ve) cells. $P$-values were determined using the two-tails Student's t-test. ${ }^{*} P<0.05$; ${ }^{* \star} P<0.01 ;{ }^{* \star *} P<0.001$. (e) Cleaved PARP and NIS immunoblot in HepG2 cells transfected with the pcDNA-NIS expression vector. $\alpha$-Actin protein levels were used to normalize equal loadings from lysate samples. Histograms represent the means of three independent experiments. Bar, S.D.

a substantial decrease of the percentage of both early (annexin $\mathrm{V}+$ ve $\mathrm{PI}-\mathrm{ve}$ ) and late (annexin $\mathrm{V}+\mathrm{ve}, \mathrm{PI}+\mathrm{ve}$ ) apoptotic cells, but not of necrotic cells (annexV - ve PI + ve) (Figure 5d). Conversely, when HepG2 cells were transfected with a NIS expression vector we could show an accumulation of cleaved PARP (Figure 5e), caspase 7 and caspase 3 (Supplementary Figure S10) levels. Together, these results indicate that NIS contributes to DNA damageinduced apoptosis in liver cancer cells and would suggest that NIS has a role in DNA damage-induced apoptotic cell death.

\section{Discussion}

NIS is expressed in many human cancers, such as differentiated thyroid cancers, breast cancer, CCAs and hepatocellular carcinoma. ${ }^{3,4,5,9}$ In thyroid cancers, NIS expression is largely controlled by the thyroid-selective transcription factors Pax-8 and Nkx2.1, which target the upstream enhancer (NUE) ( -9470 and -9046 relative to the ATG), ${ }^{19,20}$ and by the cardiac homeobox transcription factor Nkx2.5, ${ }^{34}$ which regulates the activity of the NIS core promoter ( -475 and -393$)$. The transcription factors and the signaling pathways that control NIS expression in 
non-thyroid cancers are still largely unknown. To gain insight into this issue, we investigated the modulation of NIS expression by, on one hand, the p53 and p53-related transcription factors in liver cancer cells and $\mathrm{PHHs}$, and, on the other hand, the DNA-damaging drug doxorubicin, which is known to induce an accumulation of, and activate, the p53-family proteins in a variety of cell types. ${ }^{40}$

The potential role of p53-family members in the regulation of NIS expression was first suggested by the demonstration that $N I S$ is a direct target gene modulated by p63 in keratinocytes ${ }^{39}$ and by in silico analyses bringing to light two putative regulatory clusters of p53REs bound by the all the p53-family members (i.e., p53, p63 and p73) in the NIS regulatory region. We found that NIS is expressed in $\mathrm{PHH}$ and overexpressed in CCA and HCC cell lines, except for the Hep3B cells, that are p53 null and do not express p73. ${ }^{38,41,42}$ Loss of p53 function is closely associated with the development of hepatocellular carcinoma (HCC) and CCA. ${ }^{43,44}$ In HCCs, the mutation frequency of the p53 gene is of $30 \%$ on average, but ranges from 0 to $67 \%$ depending on the geographical area and the etiological factors. ${ }^{45,46}$ In CCAs, the p53 gene mutation frequency also shows a high variability with reported values ranging from 20 to $61 \% .^{47,48}$ TP63 and TP73 are only rarely mutated but are often deregulated with an overexpression of DTA- and DN-dominant negative and transactivation-deficient $p 73$ isoforms generated, respectively, by alternative splicing from the $\mathrm{P} 1 \mathrm{p} 73$ promoter or usage of the alternative internal P2p73 promoter. ${ }^{49-51}$ TP63 mRNA and protein levels are also detected in liver cells and HCC cell lines. ${ }^{52}$ Immunohistochemical studies have also show that p63 expression is upregulated in CCAs, especially poorly differentiated ones. ${ }^{53}$

By combining reporter luciferase assays, endogenous mRNA expression analysis, chromatin immunoprecipitation experiments and siRNA-mediated silencing, we have shown that NIS expression is controlled by the p53-family members in normal and transformed hepatocytes. ChIP analysis showed that the two clusters of p53-responsive elements we identified in the NIS proximal promoter are both functional and are occupied differentially by the p53-family proteins to regulate the basal and doxorubicin-induced transcription of NIS. A correlation between p53 inactivation, thyroid cancer aggressiveness and lack of differentiation markers, including NIS, has long been noted. ${ }^{54,55}$ However, whether NIS is a target gene for p53-family members in the context of thyroid cancer remains to be determined. Interestingly, in $\mathrm{PHHs}$, the binding of the p53-family members to the NIS promoter is low in the basal state and only modestly augmented by a doxorubicin treatment, which, consequently, fails to induce any significant NIS expression. The observed differences in the regulation of NIS gene expression between normal and cancer liver cells likely reflects the complexity of the p53 family, which includes a large number of isoforms that can have opposite transcriptional effects in different cells and cell states. Whether similar differences in p53-dependent NIS gene activation between normal and cancer cells also exist in tissues other than the liver remains to be investigated.

The role of the p53-family members as tumor suppressors as well as effectors of cell cycle arrest and cell death in response to DNA damage is well recognized. ${ }^{40,56}$ Here we show that, in response to doxorubicin, p73 and p53 are actively recruited to the NIS promoter leading to an increase of endogenous NIS expression and detection of the NIS protein at the cell surface. ChIP analysis also showed that the two clusters of p53RE in the NIS promoter are used differentially to regulate basal and doxorubicin-induced NIS transcription by the p53-family members.

To evaluate whether the p53-mediated NIS gene induction contributed to cellular responses following DNA damage, we modulated NIS levels either by specific siRNA or by transfection of a NIS expression vector in HepG2 cells exposed to doxorubicin. We found that silencing NIS expression decreased doxorubicin-induced apoptosis in liver cancer cells, and, conversely, overexpressed NIS favors PARP cleavage and apoptosis, pointing to a possible, as-yet unsuspected, role of NIS accumulation in apoptotic cell death.

Altogether, our results unveil a complex transcriptional network involving the p53 family, and suggest that the modulation of NIS by DNA-damaging agents is potentially exploitable to boost NIS upregulation in vivo. NIS-mediated iodide accumulation within thyroid cell is the basis for thyroid nodules scintigraphic evaluation of radioiodine-based treatment of post-surgical residual, recurrent or metastatic disease in thyroid cancer. Significant efforts have been made to exploit NIS for developing radioiodine-based diagnostic and therapeutic procedures in non-thyroid malignant diseases has been reviewed. ${ }^{20}$ In non-thyroid tumors, NIS expression is generally lower than in thyroid cancers and NIS protein is prevalently, if not exclusively, cytoplasmic. ${ }^{19,20}$ Nevertheless, some NIS mRNA expressing breast cancers display iodine scintigraphic uptake. ${ }^{11}$ A selective induction of NIS in tumor cells might be instrumental for developing NIS-mediated radionuclide therapy and represent an attractive alternative to NIS DNA-mediated transduction. Our finding that doxorubicin induced NIS protein selective accumulation in liver cancer cells might suggests to consider a combination of doxorubicin and radioiodine therapy against NIS-expressing non-thyroid cancers.

\section{Materials and Methods}

Cell lines and DNA transfection. Human hepatoma Hep3B, HepG2, HuH7 and CCA CCSW1 and CCLP1 cell lines were cultured in Dulbecco's modified Eagle's medium with 10\% fetal bovine serum. PHHs were prepared from two adult patients undergoing lobectomy or segmental liver resection for medically required purposes unrelated to this research program. FT304 was a 52-year-old female transplant donor and FT310, a 60-year-old female, undewent liver resection for a cystadenoma. Both patients were negative for HBV, HCV and HIV. The two patients gave their written informed consent and the use of the their liver specimens for scientific purposes was approved by the French National Ethic Committee. $\mathrm{PHH}$ were prepared and cultured as described elsewhere. ${ }^{57} \mathrm{PHH}$ were plated into collagen-coated dishes (BD Biosciences, Franklin Lakes, NJ, USA) at $1.7 \times 10^{5}$ cells $/ \mathrm{cm}^{2}$ in a hormonally and chemically defined medium. ${ }^{57}$

Cells transfections with the indicated luciferase reporters, expression vectors encoding p53-family members or NIS and Renilla luciferase $\mathrm{pRL}$ null vector were performed using TransIT-TKO and TransIT-LT1 reagents from Mirus (Mirus Bio LLC, Madison, WI, USA). When indicated, $24 \mathrm{~h}$ after transfection cells were treated with Doxorubicin (Sigma-Aldrich, St Louis, MO, USA) for additional $24 \mathrm{~h}$. Cell lysates were assayed for luciferase activity using the dual-luciferase assay system (Promega, Fitchburg, WI, USA).

Antibodies, plasmids, siRNAs and chemicals. The following antibodies were used: anti-p53 (D01) mouse monoclonal, anti-p63 (4A4) rabbit polyclonal from Santa Cruz Biotechnology Inc. (San Diego, CA, USA); anti-p73 mouse monoclonal (IMG-259A) from Imgenex (San Diego, CA, USA), anti-cleaved PARP rabbit polyclonal from Cell Signaling Technology (Danvers, MA, USA); 
anti-NIS LP10 rabbit polyclonal. ${ }^{9,58}$ pcDNA-HA-P53, pcDNA-HA-P63 $\alpha$, pcDNAHA-P73 $\alpha$ expression vectors are transfected into cells using TransIT-TKO and TransIT-LT1 reagents from Mirus (Mirus Bio LLC). ${ }^{59}$ pcDNA-NIS expression vectors are described elsewhere. ${ }^{18}$ The NIS promoter luciferase reporter construct p2.0-NIS-luc was described by Ryu. ${ }^{60}$ Double-stranded Smart Pool siRNA specific for human NIS, p53, TAp63, TAp73 and control siRNA were purchased from Dharmacon Research (Lafayette, CO, USA) and transfected into cells using TransITTKO and TransIT-LT1 reagents from Mirus (Mirus Bio LLC). Doxorubicin (D1515) was purchased from Sigma (Sigma-Aldrich).

Immmunoblotting. Protein extracts were prepared in RIPA buffer (10 mM Tris- $\mathrm{HCl}$, pH 7.5, $137 \mathrm{mM} \mathrm{NaCl}, 1 \mathrm{mM}$ EDTA, $0.5 \mathrm{mM}$ EGTA, 1\% Nonidet P-40, $0.1 \%$ SDS, $0.1 \%$ sodium deoxycholate, $1 \%$ Triton X-100) containing protease inhibitors. Cell surface proteins were isolated using the Pierce Cell Surface Protein Isolation Kit (Thermo Fisher Scientific Inc., Waltham, MA, USA). Samples were analyzed by electrophoresis with Tris-acetate or Bis-Tris minigels (NuPAGE). Equal amounts of protein extracts were separated on 10\% SDS-PAGE, transferred onto nitrocellulose (GE-Amersham, Healthcare Life Sciences, Uppsala, Sweden) and processed for immunoblot using HRP-conjugated secondary antibodies Santa Cruz Biotechnology Inc. and chemoluminescence (GE-Amersham).

Quantitative RT-PCR. Total RNAs were isolated using Trizol reagent (Invitrogen, Carlsbad, CA, USA) and then reverse transcribed into CDNA using a Revert Aid First Strand cDNA Synthesis Kit (Thermo Fisher Scientific Inc.,), amplified and quantified by detection of SYBR Green (Roche Diagnostics, Indianapolis, IN, USA). They were quantified by nano-drop and their quality was assessed by electrophoresis. Real-time PCR and melting curve analysis were done in a LightCycler 3.0 (Roche Diagnostics) using SYBR Green master mix (Roche Diagnostics). The following primers were purchased from (Invitrogen): NIS, forward (fwd) (5'-CTTCTGAACTCGGTCCTCACAC- $\left.3^{\prime}\right)$ and reverse (rev) (5'-TCCAGAATGTATAGCGGCTC-3').

ChIP assay. Protein complexes were cross-linked to DNA in living nuclei by adding $1 \%$ of formaldehyde (Merck, Whitehouse Station, NJ, USA) to the culture medium for $10 \mathrm{~min}$ at room temperature. Crosslinking was stopped by the addition of $0.125 \mathrm{M}$ glycine. Cross linked cells were scraped, washed with PBS. Cells were pelleted by centrifugation and lysed in nuclear lysis buffer ( $1 \%$ SDS, $10 \mathrm{mM}$ EDTA, $50 \mathrm{mM}$ Tris-Hcl pH 8.1, $0.5 \mathrm{mM}$ phenylmethylsulfonyl fluoride PMSF, $100 \mathrm{ng} / \mathrm{ml}$ leupeptin, $100 \mathrm{ng} / \mathrm{ml}$ aprotinin). The resulting chromatin solution was sonicated for 10 pulses of $45 \mathrm{~s}$ at high power (Bioruptor sonicator, Diagenode, Liege, Belgium) to generate $500-1500 \mathrm{bp}$ DNA fragments. After microcentrifugation, the supernatant was precleared with blocked protein G plus (Pierce, Rockford, IL, USA) diluted $1: 10$ with dilution buffer $(0.01 \%$ SDS, $1.1 \%$ Triton X-100, 1.2 mM EDTA, $16.7 \mathrm{mM}$ Tris-Hcl pH 8.1, $167 \mathrm{mM} \mathrm{NaCl}, 0.5 \mathrm{mM}$ PMFS, $100 \mathrm{ng} / \mathrm{ml}$ leupeptin, $100 \mathrm{ng} / \mathrm{ml}$ aprotinin), and divided into aliquots. Five micrograms of antibody was added to each aliquot of chromatin and incubated on a rotating platform for $12-16 \mathrm{~h}$ at $4{ }^{\circ} \mathrm{C}$. Antibody-protein-DNA complexes were isolated by immunoprecipitation with blocked protein $\mathrm{G}$ plus. Following extensive washings, ChIPed DNA was analyzed by qPCR using SYBR Green master mix (Roche Diagnostics) in a LightCycler480 (Roche) with the following primer pairs: Primer A- FOR 5'-CCTGAGATGACAGCTCGTTGG-3'; primer A-REV 5'-AATGGGACGTGGTAA CGAAAGC-3'; primer B -FOR 5'-GGAGCCCAATAAATCTGCAA-3'; primer B- REV 5'-GTC TGTCTGTCCTGCCACCT-3'.

Flow cytometry. Primary antibody staining was performed by incubating cells for $45 \mathrm{~min}$ on ice with $10 \mathrm{ug} / \mathrm{ml}$ of LP10 polyclonal rabbit anti-NIS. After extensive washing, secondary FITC-conjugated goat anti-rabbit antibody (Invitrogen) was added and cells were incubated for further $30 \mathrm{~min}$ on ice. All incubation and washing steps were performed in PBS with $2 \%$ FBS. Data were acquired on a FACSCanto (Becton Dickinson, Franklin Lakes, NJ, USA) and analyzed with FlowJo software (Treestar Inc, Ashland, OR, USA, version 8.8.7) and Prism software (GraphPad, Software Inc, La Jolla, CA, USA, version 4). NIS relative expression in each sample was calculated as the ratio between the median fluorescent intensity of sample stained with both primary and secondary Abs, and the MFI of sample stained with secondary Ab alone.

\section{Conflict of Interest}

The authors declare no conflict of interest.
Acknowledgements. We thank J Hervé, N Moniaux (INSERM U785, Villejuif) and J Clerc (Paris Descartes University) for insightful discussions; P Pineau (Pasteur Institute, Paris) for providing liver cancer cell lines. This work was supported by Grants from INSERM, Association pour la Recherche contre le Cancer (ARC 3555, ARC 4866; to JF) and Institut National du Cancer (INCa PL027, INCa 2009-PAIR-CHC; to JF); Associazione Italiana per la Ricerca sul Cancro (AIRC) to ML; MIUR-Cofin and Progetti di Ateneo, Sapienza University of Rome to ML. FG has been supported by fellowships from the Fondazione A. Cesalpino and Rome Oncogenomic Center at the Regina Elena Cancer Institute.

1. Carrasco N. lodide transport in the thyroid gland. Biochim Biophys Acta 1993; 1154: 65-82.

2. Dai G, Levy $\mathrm{O}$, Carrasco N. Cloning and characterization of the thyroid iodide transporter. Nature 1996; 379: 458-460.

3. Perron B, Rodriguez AM, Leblanc G, Pourcher T. Cloning of the mouse sodium iodide symporter and its expression in the mammary gland and other tissues. J Endocrinol 2001; 170: $185-196$.

4. Spitzweg C, Joba W, Eisenmenger W, Heufelder AE. Analysis of human sodium iodide symporter gene expression in extrathyroidal tissues and cloning of its complementary deoxyribonucleic acids from salivary gland, mammary gland, and gastric mucosa. J Clin Endocrinol Metab 1998; 83: 1746-1751.

5. Wapnir IL, van de Rijn M, Nowels K, Amenta PS, Walton K, Montgomery K et al. Immunohistochemical profile of the sodium/iodide symporter in thyroid, breast, and other carcinomas using high density tissue microarrays and conventional sections. J Clin Endocrinol Metab 2003; 88: 1880-1888.

6. Hingorani M, Spitzweg C, Vassaux G, Newbold K, Melcher A, Pandha H et al. The biology of the sodium iodide symporter and its potential for targeted gene delivery. Curr Cancer Drug Targets 2010; 10: 242-267.

7. Mazzaferri EL, Kloos RT. Current approaches to primary therapy for papillary and follicular thyroid cancer. J Clin Endocrinol Metab 2001; 86: 1447-1463.

8. Altorjay A, Dohan O, Szilagyi A, Paroder M, Wapnir IL, Carrasco N. Expression of the $\mathrm{Na}+/$ - symporter (NIS) is markedly decreased or absent in gastric cancer and intestinal metaplastic mucosa of Barrett esophagus. BMC Cancer 2007; 7: 5.

9. Liu B, Herve J, Bioulac-Sage P, Valogne Y, Roux J, Yilmaz F et al. Sodium iodide symporter is expressed at the preneoplastic stages of liver carcinogenesis and in human cholangiocarcinoma. Gastroenterology 2007; 132: 1495-1503.

10. Tazebay UH, Wapnir IL, Levy O, Dohan O, Zuckier LS, Zhao QH et al. The mammary gland iodide transporter is expressed during lactation and in breast cancer. Nat Med 2000; 6 : $871-878$.

11. Knostman KA, McCubrey JA, Morrison CD, Zhang Z, Capen CC, Jhiang SM. PI3K activation is associated with intracellular sodium/iodide symporter protein expression in breast cancer. BMC Cancer 2007; 7: 137.

12. Willhauck MJ, Sharif-Samani B, Senekowitsch-Schmidtke R, Wunderlich N, Goke B, Morris $\mathrm{JC}$ et al. Functional sodium iodide symporter expression in breast cancer xenografts in vivo after systemic treatment with retinoic acid and dexamethasone. Breast Cancer Res Treat 2008; 109: 263-272.

13. Ryan J, Curran CE, Hennessy E, Newell J, Morris JC, Kerin MJ et al. The sodium iodide symporter (NIS) and potential regulators in normal, benign and malignant human breast tissue. PLoS One 2011; 6: e16023.

14. Kogai T, Liu YY, Mody K, Shamsian DV, Brent GA. Regulation of sodium iodide symporter gene expression by Rac1/p38beta mitogen-activated protein kinase signaling pathway in MCF-7 breast cancer cells. J Biol Chem 2012; 287: 3292-3300.

15. Moon DH, Lee SJ, Park KY, Park KK, Ahn SH, Pai MS et al. Correlation between $99 \mathrm{mTc}$-pertechnetate uptakes and expressions of human sodium iodide symporter gene in breast tumor tissues. Nucl Med Biol 2001; 28: 829-834.

16. Cianfarani F, Baldini E, Cavalli A, Marchioni E, Lembo L, Teson M et al. TSH receptor and thyroid-specific gene expression in human skin. J Invest Dermatol 2010; 130: 93-101.

17. Damle AA, Narkar AA, Badwe RA. Radioiodide uptake and sodium iodide symporter expression in breast carcinoma. Indian J Exp Biol 2011; 49: 416-422.

18. Lacoste C, Hervé J, Bou Nader M, Dos Santos A, Moniaux N, Valogne $Y$ et al. lodide transporter NIS regulates cancer cell motility and invasiveness by interacting with the Rho guanine nucleotide exchange factor LARG. Cancer Res 2012; 72: 5505-5515.

19. Kogai T, Brent GA. The sodium iodide symporter (NIS): Regulation and approaches to targeting for cancer therapeutics. Pharmacol Ther 2012; 135: 355-370.

20. Kogai T, Taki K, Brent GA. Enhancement of sodium/iodide symporter expression in thyroid and breast cancer. Endocr Relat Cancer 2006; 13: 797-826.

21. De Felice M, Di Lauro R. Thyroid development and its disorders: genetics and molecular mechanisms. Endocr Rev 2004; 25: 722-746.

22. Ohno M, Zannini M, Levy O, Carrasco N, di Lauro R. The paired-domain transcription factor Pax8 binds to the upstream enhancer of the rat sodium/iodide symporter gene and participates in both thyroid-specific and cyclic-AMP-dependent transcription. Mol Cell Biol 1999; 19: 2051-2060.

23. Taki K, Kogai T, Kanamoto Y, Hershman JM, Brent GA. A thyroid-specific far-upstream enhancer in the human sodium/iodide symporter gene requires Pax-8 binding and cyclic adenosine 3',5'-monophosphate response element-like sequence binding proteins for full 
activity and is differentially regulated in normal and thyroid cancer cells. Mol Endocrinol 2002; 16: 2266-2282.

24. Puppin C, Arturi F, Ferretti E, Russo D, Sacco R, Tell G et al. Transcriptional regulation of human sodium/iodide symporter gene: a role for redox factor-1. Endocrinology 2004; 145 1290-1293

25. Smith VE, Franklyn JA, McCabe CJ. Expression and function of the novel proto-oncogene PBF in thyroid cancer: a new target for augmenting radioiodine uptake. J Endocrinol 2011; 210: $157-163$

26. Xu J, Kogai T, Brent GA, Hershman JM. A GC box in the human sodium iodide symporter gene promoter is essential for full activity. Thyroid 2002; 12: 107-114.

27. Schmutzler C, Schmitt TL, Glaser F, Loos U, Kohrle J. The promoter of the human sodium iodide-symporter gene responds to retinoic acid. Mol Cell Endocrinol 2002; 189: 145-155.

28. Kogai T, Kanamoto Y, Li Al, Che LH, Ohashi E, Taki K et al. Differential regulation of sodium/iodide symporter gene expression by nuclear receptor ligands in MCF-7 breast cancer cells. Endocrinology 2005; 146: 3059-3069.

29. Kogai T, Schultz JJ, Johnson LS, Huang M, Brent GA. Retinoic acid induces sodium/iodide symporter gene expression and radioiodide uptake in the MCF-7 breast cancer cell line. Proc Natl Acad Sci USA 2000; 97: 8519-8524.

30. Tanosaki S, Ikezoe T, Heaney A, Said JW, Dan K, Akashi M et al. Effect of ligands of nuclear hormone receptors on sodium/iodide symporter expression and activity in breast cancer cells. Breast Cancer Res Treat 2003; 79: 335-345.

31. Ohashi E, Kogai T, Kagechika H, Brent GA. Activation of the PI3 kinase pathway by retinoic acid mediates sodium/iodide symporter induction and iodide transport in MCF-7 breast cancer cells. Cancer Res 2009; 69: 3443-3450.

32. Alotaibi H, Yaman E, Salvatore D, Di Dato V, Telkoparan P, Di Lauro R et al. Intronic elements in the $\mathrm{Na}+/$ l- symporter gene (NIS) interact with retinoic acid receptors and mediate initiation of transcription. Nucleic Acids Res 2010; 38: 3172-3185.

33. Willhauck MJ, O Kane DJ, Wunderlich N, Goke B, Spitzweg C. Stimulation of retinoic acidinduced functional sodium iodide symporter (NIS) expression and cytotoxicity of (1)(3)(1) by carbamazepine in breast cancer cells. Breast Cancer Res Treat 2011; 125: 377-386.

34. Dentice M, Luongo C, Elefante A, Romino R, Ambrosio R, Vitale M et al. Transcription factor Nkx-2.5 induces sodium/iodide symporter gene expression and participates in retinoic acid- and lactation-induced transcription in mammary cells. Mol Cell Biol 2004; 24 $7863-7877$.

35. Lin Y, Shi CY, Li B, Soo BH, Mohammed-Ali S, Wee A et al. Tumour suppressor p53 and $\mathrm{Rb}$ genes in human hepatocellular carcinoma. Ann Acad Med Singapore 1996; 25: 22-30.

36. Hsu IC, Tokiwa T, Bennett W, Metcalf RA, Welsh JA, Sun T et al. p53 gene mutation and integrated hepatitis B viral DNA sequences in human liver cancer cell lines. Carcinogenesis 1993; 14: 987-992

37. Caca K, Feisthammel J, Klee K, Tannapfel A, Witzigmann H, Wittekind C et al. Inactivation of the INK4a/ARF locus and p53 in sporadic extrahepatic bile duct cancers and bile tract cancer cell lines. Int J Cancer 2002; 97: 481-488.

38. Pineau P, Marchio A, Nagamori S, Seki S, Tiollais P, Dejean A. Homozygous deletion scanning in hepatobiliary tumor cell lines reveals alternative pathways for liver carcinogenesis. Hepatology 2003; 37: 852-861.

39. Testoni B, Borrelli S, Tenedini E, Alotto D, Castagnoli C, Piccolo S et al. Identification of new p63 targets in human keratinocytes. Cell Cycle 2006; 5: 2805-2811.

40. Lunghi P, Costanzo A, Mazzera L, Rizzoli V, Levrero M, Bonati A. The p53 family protein p73 provides new insights into cancer chemosensitivity and targeting. Clin Cancer Res 2009; 15: 6495-6502.

41. Hageman J, Eggen BJ, Rozema T, Damman K, Kampinga HH, Coppes RP. Radiation and transforming growth factor-beta cooperate in transcriptional activation of the profibrotic plasminogen activator inhibitor-1 gene. Clin Cancer Res 2005; 11: 5956-5964.

42. Lasfer M, Davenne L, Vadrot N, Alexia C, Sadji-Ouatas Z, Bringuier AF et al. Protein kinase PKC delta and C-Abl are required for mitochondrial apoptosis induction by genotoxic stress in the absence of p53, p73 and Fas receptor. FEBS Lett 2006; 580: 2547-2552.

43. Imbeaud S, Ladeiro Y, Zucman-Rossi J. Identification of novel oncogenes and tumor suppressors in hepatocellular carcinoma. Semin Liver Dis 2010; 30: 75-86.
44. Nault JC, Zucman-Rossi J. Genetics of hepatobiliary carcinogenesis. Semin Liver Dis 2011; 31: 173-187.

45. Woo HG, Wang XW, Budhu A, Kim YH, Kwon SM, Tang ZY et al. Association of TP53 mutations with stem cell-like gene expression and survival of patients with hepatocellular carcinoma. Gastroenterology 2011; 140: 1063-1070.

46. Villanueva A, Hoshida Y. Depicting the role of TP53 in hepatocellular carcinoma progression. J Hepatol 2011; 55: 724-725.

47. Tannapfel A, Weinans L, Geissler F, Schutz A, Katalinic A, Kockerling F et al. Mutations of p53 tumor suppressor gene, apoptosis, and proliferation in intrahepatic cholangiocellular carcinoma of the liver. Dig Dis Sci 2000; 45: 317-324.

48. Xiaofang L, Kun T, Shaoping Y, Zaiqiu W, Hailong S. Correlation between promoter methylation of p14(ARF), TMS1/ASC, and DAPK, and p53 mutation with prognosis in cholangiocarcinoma. World J Surg Oncol 2012; 10: 5.

49. Muller M, Schilling T, Sayan AE, Kairat A, Lorenz K, Schulze-Bergkamen H et al. TAp73/ Delta Np73 influences apoptotic response, chemosensitivity and prognosis in hepatocellular carcinoma. Cell Death Differ 2005; 12: 1564-1577.

50. Castillo J, Goni S, Latasa MU, Perugorria MJ, Calvo A, Muntane J et al. Amphiregulin induces the alternative splicing of p73 into its oncogenic isoform DeltaEx2p73 in human hepatocellular tumors. Gastroenterology 2009; 137: 1805-1815.

51. Mundt HM, Stremmel W, Melino G, Krammer PH, Schilling T, Muller M. Dominant negative (DeltaN) p63alpha induces drug resistance in hepatocellular carcinoma by interference with apoptosis signaling pathways. Biochem Biophys Res Commun 2010; 396: 335-341

52. Petitjean A, Cavard C, Shi H, Tribollet V, Hainaut P, Caron de Fromentel C. The expression of TA and DeltaNp63 are regulated by different mechanisms in liver cells. Oncogene 2005; 24: $512-519$

53. Ramalho FS, Ramalho LN, Della Porta L, Zucoloto S. Comparative immunohistochemical expression of p63 in human cholangiocarcinoma and hepatocellular carcinoma. J Gastroenterol Hepatol 2006; 21: 1276-1280.

54. Donghi R, Longoni A, Pilotti S, Michieli P, Della Porta G, Pierotti MA. Gene p53 mutations are restricted to poorly differentiated and undifferentiated carcinomas of the thyroid gland. J Clin Invest 1993; 91: 1753-1760.

55. Fagin JA, Tang SH, Zeki K, Di Lauro R, Fusco A, Gonsky R. Reexpression of thyroid peroxidase in a derivative of an undifferentiated thyroid carcinoma cell line by introduction of wild-type p53. Cancer Res 1996; 56: 765-771.

56. Flores ER, Sengupta S, Miller JB, Newman JJ, Bronson R, Crowley D et al. Tumor predisposition in mice mutant for p63 and p73: evidence for broader tumor suppressor functions for the p53 family. Cancer Cell 2005; 7: 363-373.

57. Pichard-Garcia L, Gerbal-Chaloin S, Ferrini JB, Fabre JM, Maurel P. Use of long-term cultures of human hepatocytes to study cytochrome P450 gene expression. Methods Enzymol 2002; 357: 311-321.

58. Herve J, Cunha AS, Liu B, Valogne Y, Longuet M, Boisgard R et al. Internal radiotherapy of liver cancer with rat hepatocarcinoma-intestine-pancreas gene as a liver tumor-specific promoter. Hum Gene Ther 2008; 19: 915-926.

59. Belloni L, Moretti F, Merlo P, Damalas A, Costanzo A, Blandino G et al. DNp73alpha protects myogenic cells from apoptosis. Oncogene 2006; 25: 3606-3612.

60. Ryu KY, Tong Q, Jhiang SM. Promoter characterization of the human $\mathrm{Na}+/ /$ - symporter. $J$ Clin Endocrinol Metab 1998; 83: 3247-3251.

(c) (i) $(9) \Theta$ Cell Death and Disease is an open-access journal published by Nature Publishing Group. This work is licensed under a Creative Commons Attribution-NonCommercialNoDerivs 3.0 Unported License. To view a copy of this license, visit http://creativecommons.org/licenses/by-nc-nd/3.0/

\section{Supplementary Information accompanies this paper on Cell Death and Disease website (http://www.nature.com/cddis)}

